\title{
Evaluation of Additive Manufacturing Processes in Fabrication of Personalized Robot
}

\author{
Shushu Wang, Rakshith Badarinath, El-Amine Lehtihet, and Vittaldas Prabhu \\ Penn State University, University Park, PA, U.S.A. \\ gloriawongtegmail.com
}

\begin{abstract}
Customers increasingly participate in the design stage of creating personalized products. Additive manufacturing (aka 3D printing) has become a popular enabler of personalization. In this paper we evaluate fabrication of an open source robot arm in terms of cost, build time, dimensional and locational accuracy, and mechanical properties. The mechanical components of the table-top robot were fabricated using two different AM processes: Fused deposition modelling (FDM) and Material Jetting (Polymer Jetting). Reducing the infill density to $50 \%$ in the FDM process resulted in a slight decrease in building time, material cost and tensile strength, and caused a 95\% drop in yield strength. Simulation of the robot's mechanical assembly using its CAD model based on the expected tolerances of the components, resulted in estimation of the end-effector positioning accuracy to be 0.01 to $0.22 \mathrm{~mm}$.
\end{abstract}

Keywords: Personalization· Fused Deposition Modelling · Material Jetting · Infill density robot $\cdot$ Dimensional and locational accuracy $\cdot$ Tensile strength

\section{$1 \quad$ Introduction}

In recent decades, companies have tried a new strategy called mass production to provide broad provision of personalized products and services (Davis, 1989), and the strategy is considered as an important competitive advantage (Fiore et al., 2003, Salvador, 2009). Kumar A (2007) points out that personalization of products and services has been put forward as a business strategy to expand the market share for the past twenty years. 3D printing enables people to fabricate things as per their preferences and serves as an alternative to purchase mass-produced goods.

3D printing creates physical products from a computer generated design file by building up each layer on top of other to produce the final part. In this work, two 3D printing techniques were used: Fused Deposition Modeling (FDM) and PolyJet 3D printing. The FDM machine extrudes and deposits a semi-molten thermoplastic filament in a crisscross manner layer by layer from the bottom up. PolyJet 3D printing, being relatively new, jets UV curable liquid photopolymer onto the building platform (PolyJet Technology, Stratasys).

A number of studies on 3D printing process characteristics have been carried out. Generally, benchmark parts, instead of functional products, are tested in these studies.

adfa, p. 1, 2011.

(C) Springer-Verlag Berlin Heidelberg 2011 
Mahesh et al. (2004) developed specific part design for benchmark tests and evaluated abilities of different AM processes based the test parts. Kim and Oh (2008) performed quantitative comparisons of mechanical properties, accuracy, roughness, speed, and material cost of several rapid prototyping processes, such as stereo lithography (SL), FDM, PolyJet, and selective laser sintering (SLS). Baich and Manogharan (2015) investigated the relationship between infill density and the resulting mechanical properties, production cost and time of FDM process. 3D Matter website (2015) featured an article on influence of infill rate, layer height and infill pattern on mechanical performance for the FDM process involving PLA material.

The objective of this paper is to evaluate the characteristics of different 3D printing machines and processes in context to fabrication of a personalized robot. A personalization case study was conducted based on MeArm $®$, an open-source desktop robot arm. It is a 4-axis pick and place robot arm controlled by a joystick. The robot parts are laser cut from acrylic sheets and assembled with M3 self-tapping screws.

The paper is organized as follows. Section 2 provides insight on the linkage mechanism of the robot arm, 3D printing process parameters settings and finite element analysis (FEA) simulation constraints and results. Section 3 compares the parts printed from different 3D printing machines in terms of material cost, building time, dimensional accuracy, assembly accuracy, and part strength. Accuracy and strength of the original MeArm parts were calculated as well. Section 4 concludes the research and puts forth future research opportunities.

\section{Case Study-3D Printing of a Desktop Robot Arm}

Eight linkage parts from MeArm robot were 3D printed using three different machines that are available in Penn State: Makerbot Replicator (5th Generation), Fortus $250 \mathrm{mc}$, and Object30 Prime, and corresponding materials are Polylactic acid (PLA), Acrylonitrile butadiene styrene (ABS), and Rigid Opaque photopolymers (VeroBlue RGD 840). To evaluate the quality 3D printed parts: (1) building time and material cost was evaluated; (2) Hole diameter at each functional robot joint, distance between holes, and part thickness was measured; (3) Assembly tolerances were calculated; (4) Tensile tests were conducted to evaluate part strength. The evaluation results were compared with the results of tests conducted on original parts from MeArm Robotics.

\subsection{Specimens Fabrication}

A 3D CAD model of MeArm linkage was built using SolidWorks and 3D printed as shown in Fig. 1, and the parts highlighted in red are the driving links.

Various manufacturing parameters can be adjusted in build-preparation software. One set of linkage specimens are printed under each setting option listed in Table 1, while other parameters just following the machine default setting. 


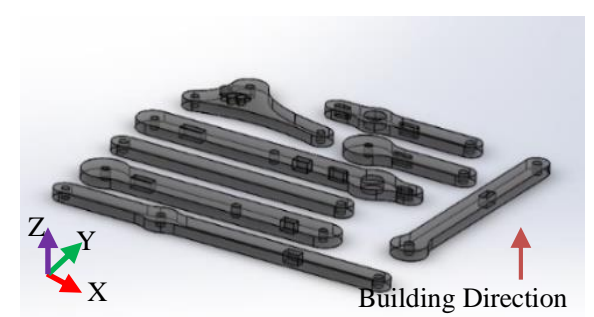

(a) $3 \mathrm{D}$ printing orientation

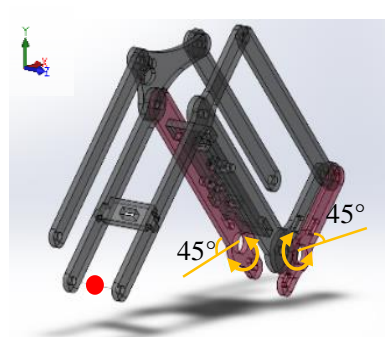

(b)Linkage 3D model

Fig. 1. Linkage parts

Table 1. Manufacturing parameters

\begin{tabular}{ccccc}
\hline Specimen & Apparatus Model & Material & $\begin{array}{c}\text { Layer } \\
\text { Thickness (mm) }\end{array}$ & $\begin{array}{c}\text { Interior } \\
\text { Density }\end{array}$ \\
\hline 1 & MakerBot & MakerBot PLA & 0.150 & $100 \%$ \\
2 & Replicator & Filament & $50 \%$ \\
\hline 3 & Fortus 250mc & ABSplus-P430 & 0.178 & $\begin{array}{c}\text { Solid } \\
\text { Sparse-low } \\
\text { density }\end{array}$ \\
4 & Object30 Prime & VeroBlue & 0.016 & - \\
\hline 5 & RGD840 & & \\
\hline
\end{tabular}

\section{$2.2 \quad$ Test Procedures}

To inspect dimensional accuracy of printedspecimens, the diameter of holes on each link were measured precisely using SmartScope Flare from Optical Gaging Products; additionally, the average value of part thickness measured using a precision caliper at 3 different points of each part is considered as the thickness of that part.

With the above measurements, an assembly simulation was conducted in SolidWorks to evaluate the positional accuracy of the gripper location. In the static FEA simulation, the servo angles were set to $45^{\circ}$ on the xz plane as shown in Fig.1(b). The red dot represents the midpoint the gripper, and thus is considered as the gripper location. It is assumed that only the linkages have dimensional errors. The errors of all the other parts in the assembly such as screws, and the tolerance of servo angles was not considered in this study.

In order to have a more comprehensive comparison of the accuracy of gripper location of the three 3D printers, a simulation with more data is carried. First, the deviations of distance between holes are assumed to follow normal distributions, and the quality requirement of $3 \mathrm{D}$ printing parts is supposed at $\pm 2 \sigma$ level based on the expected tolerance. Second, for each machine type, 50 deviations were sampled from the normal distribution for every distance value. And the sampled deviations were added to the distance for every linkage. Finally, linkages with modified distance between holes were assembled in SolidWorks. For each machine type, 50 assembly were created and the 
corresponding gripper locations were recorded. Since MakerBot does not provide estimating dimensional accuracy, this paper assumes $\pm 0.500 \mathrm{~mm}$ as the dimensional accuracy based on the work of Melenka et al. (2015). The stated dimensional accuracies of Fortus and Object $3 \mathrm{D}$ printers' is $\pm 0.241 \mathrm{~mm}$ and $\pm 0.100 \mathrm{~mm}$ respectively.

A finite element analysis (FEA) simulation is performed on the linkage mechanism in SolidWorks. The simulation is to evaluate the stress and strain of each link when robot arm is picking up a $1 \mathrm{~N}$ object (including gripper weight) with each servo produces a torque of $0.17 \mathrm{Nm}$ according to specification of the micro servo. "Fixed Hinge" was applied to the revolute joints of the robot arm. To compare the mechanical performance of 3D printing specimens and MeArm robot's acrylic parts, tensile tests were done.

\section{$3 \quad$ Results and Discussions}

\subsection{Specimens Fabrication Results}

Based on the 3D printing settings in Section 2, 5 sets of specimens were fabricated and are shown in Fig.2.

The building preparation software of 3D printers provided an estimated building time and raw material consumption. Fig. 3 provides a comparison between printed specimens in terms of building time and material cost calculated from the consumption.

Obviously, the FDM process needs a longer building time than the PolyJet process. To build solid parts, MakerBot Replicator requires a building time of about 2 times longer than Object30 Prime. Additionally, the building time of MakerBot Replicator is about $15 \%$ longer than that of Fortus $250 \mathrm{mc}$. Moreover, the reduction in infill density only results in a slight decrease in building time for the FDM process.

The PolyJet process has a higher raw material cost than the FDM process. For solid parts, the Object30 Prime costs about 6 times more than the MakerBot Replicator, and $20 \%$ more than the Fortus $250 \mathrm{mc}$. Actually, ABS has a higher unit price than Object's material. However, Object still cost more for the same parts due to its higher density. In terms of material cost, the reduction of interior density resulted in an apparent decrease of $16 \%$ for MakerBot Replicator and $21 \%$ for Fortus $250 \mathrm{mc}$.

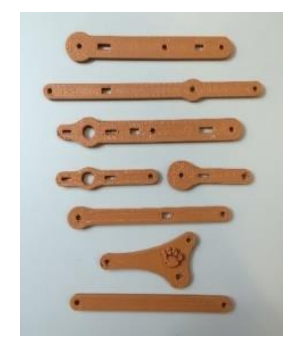

(a) MakerBot Replicator

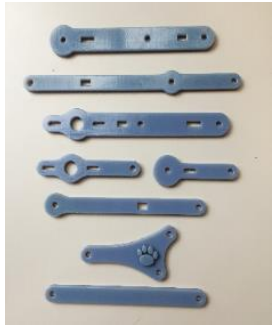

(b) Fortus $250 \mathrm{mc}$

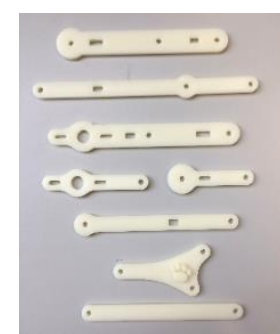

(c) Object30 Prime

Fig. 2. Specimens fabricated by $3 \mathrm{D}$ printers 


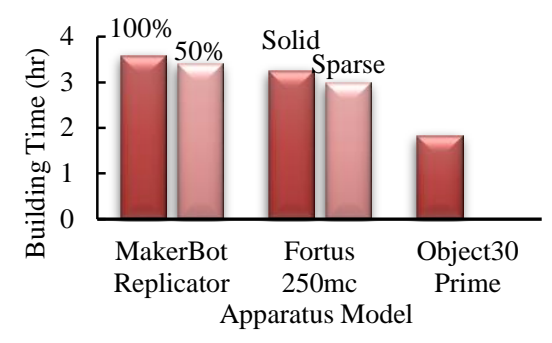

(a) Building time comparison

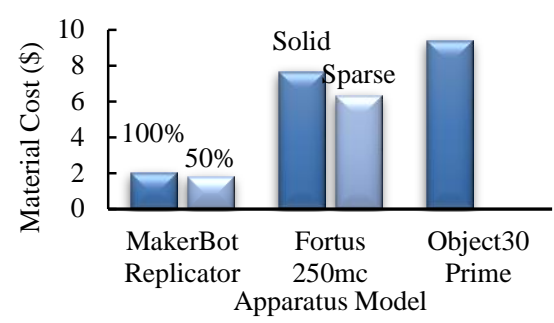

(b) Material cost comparison

Fig. 3. Comparison of apparatus models and processes

\subsection{Dimensional Accuracy and Assembly Accuracy}

To investigate dimensional accuracy, several functional dimensions were measured, including the diameter of circular holes at links, the relative location of these holes, and the thickness of parts. Fig. 4 describes deviations in these measurements, observed in the 5 sets of printed specimens and original MeArm pats.

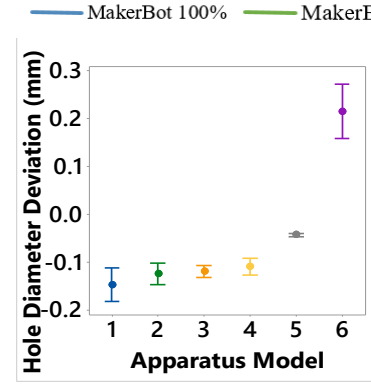

(a) Hole diameter deviation

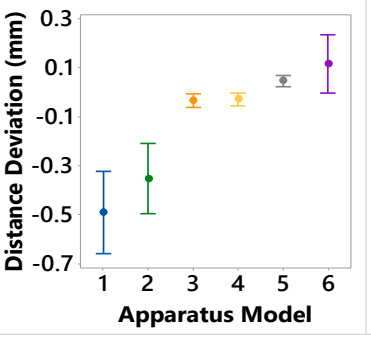

(b) Distance deviation

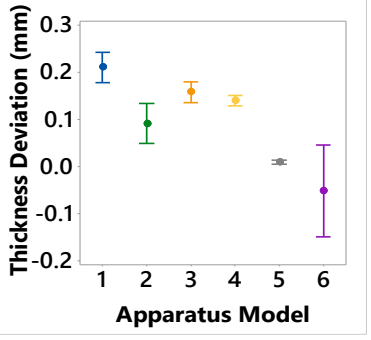

(c)Thickness deviation

Fig. 4. Dimensional deviation

Fig.4 (a) indicates that Object 30 Prime provides the best performance in hole size with the smallest deviation and variance, and MeArm parts are the worst. The holes of MeArm parts have positive deviations compared with the nominal size, which results in a clearance fits with screws to enable joints to rotate smoothly. In contrast, 3D printing process produces negative deviations because of material shrinkage after cooling. Furthermore, the difference in infill densities does not have any significant influence on dimensional accuracy. In Fig.4 (b), MakerBot Replicator makes the largest deviation and variance in distance. Fortus $250 \mathrm{mc}$ and Object30 Prime have smaller deviations. The performance of MeArm parts is between MakerBot and other two 3D printers. Fig.4 (c) shows that the Object30 Prime and MeArm have the best performance in thickness, while MakerBot Replicator is the worst. It can be concluded that the Object30 Prime has better $\mathrm{z}$ resolution and control compared to other two printers.

Based on the measurement of distance between screw holes and parts thickness, linkages are assembled in SolidWorks. Table 2 lists the deviation of gripper location of $3 \mathrm{D}$ printing specimens and the original MeArm parts. 
Table 2. Gripper location deviation

\begin{tabular}{ccc}
\hline Apparatus Model & Interior Density & Deviation $(\mathrm{XYZ})(\mathrm{mm})$ \\
\hline \multirow{2}{*}{ MakerBot Replicator } & $100 \%$ & $(0.81,0.16,-0.87)$ \\
& $50 \%$ & $(0.57,0.07,-0.42)$ \\
\hline \multirow{2}{*}{ Fortus 250mc } & Solid & $(-0.10,0.03,-0.61)$ \\
& Sparse & $(-0.04,0.02,-0.58)$ \\
\hline Object30 Prime & - & $(0.10,0.01,-0.03)$ \\
\hline MeArm Parts & - & $(-0.22,0.32,0.03)$ \\
\hline
\end{tabular}

Assembly of MeArm parts has more accurate gripper location than MakerBot parts assembly, but is worse than Fortus and Object. The assembly of Fortus parts has similar accuracy in XY plane with Object, but Object has much better $\mathrm{Z}$ axis accuracy. The reason is that Object parts have similar distance accuracy to Fortus parts but are more accurate in thickness. The MakerBot Replicator produced the largest deviation in gripper location.

Simulation result of gripper location deviations is shown in Fig.5. MakerBot Replicator has the most scatter, thus larger deviations in gripper location, and is followed by Fortus 250mc, while Object30 Prime has the smallest deviations.

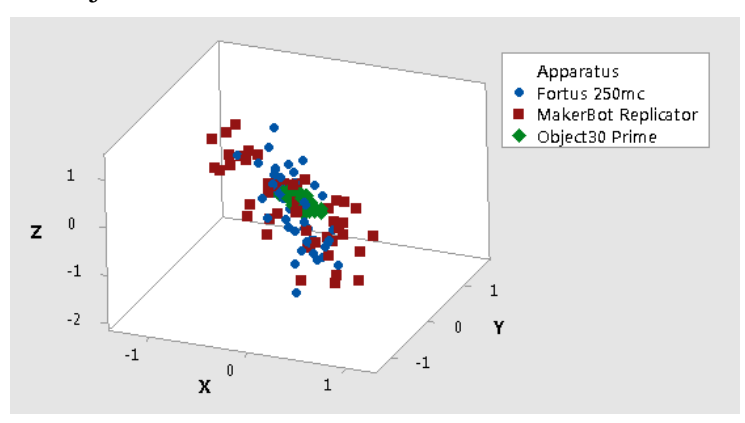

Fig. 5. Simulated XYZ deviations of gripper location

\subsection{FEA Simulation and Tensile Tests}

In the static FEA, the maximum stress and the maximum strain was observed at the driving links due to servo torque, and the simulation results of different materials are similar as shown in Table 3. Table 4 shows the tensile test results of the same part fabricated from different processes, and Fig. 6 is a comparison of stress-strain curves.

Table 3. FEA simulation results

\begin{tabular}{|c|c|c|c|c|}
\hline Strength Material & $\begin{array}{c}\text { MakerBot PLA } \\
\text { Filament }\end{array}$ & ABSplus-P430 & $\begin{array}{l}\text { VeroBlue } \\
\text { RGD840 }\end{array}$ & Acrylic Sheet \\
\hline Maximum Stress (MPa) & 7.42 & 7.41 & 7.42 & 7.42 \\
\hline Maximum Strain (\%) & 0.19 & 0.27 & 0.29 & 0.19 \\
\hline
\end{tabular}


Table 4. Tensile test results comparison

\begin{tabular}{ccccc}
\hline Material & Density & $\begin{array}{c}\text { Yield Strength } \\
(\mathrm{MPa})\end{array}$ & $\begin{array}{c}\text { Tensile Strength } \\
(\mathrm{MPa})\end{array}$ & $\begin{array}{c}\text { Break Elongation } \\
(\%)\end{array}$ \\
\hline MakerBot PLA & $100 \%$ & 22.55 & 45.31 & 4.0 \\
Filament & $50 \%$ & 1.65 & 39.14 & 3.4 \\
\hline \multirow{2}{*}{ ABSplus-P430 } & Solid & 32.13 & 32.66 & 7.5 \\
& Sparse & 1.22 & 23.79 & 6.0 \\
\hline VeroBlue RGD840 & - & 52.66 & 54.52 & 6.9 \\
\hline Acrylic Sheet & - & 1.37 & 42.87 & 2.0 \\
\hline
\end{tabular}

As stated in datasheet from MakerBot, the average tensile strength of PLA is 48MPa. 3D Matter website concluded from several experiments that the elongation at break of PLA in 3D printing is 4\%-6\%. Furthermore, according to the datasheet by Stratasys, for ABSplus-P430, the yield strength is $31 \mathrm{MPa}$; the tensile strength is $33 \mathrm{MPa}$; and elongation at break is 6\%; while for VeroBlue RGD840, the tensile strength is stated to be $50-60 \mathrm{MPa}$ and elongation at break is $6 \%$.

The tensile test results of $100 \%$ infill rate PLA, solid ABS, and VeroBlue RGD840 are similar to values stated in the material datasheets. The elongation at break of VeroBlue is only half of official data. The possible reason for this can be attribute to different specimen geometry used by Stratasys.

It can be concluded that the VeroBlue material is the best in terms of both yield strength and tensile strength. PLA has larger tensile strength than ABS, but lower yield strength. Besides, 3D printing materials are more ductile than the MeArm acrylic material. When the infill density of PLA and ABS was reduced, the tensile strength was reduced by less than $30 \%$. However, a sharp drop in the yield strength was aboserved. Therefore, interior density has a large influence on yield strength.

According to the tensile test results and FEA simulation results, 3D printing parts were evaluated to be strong enough for the robot arm.

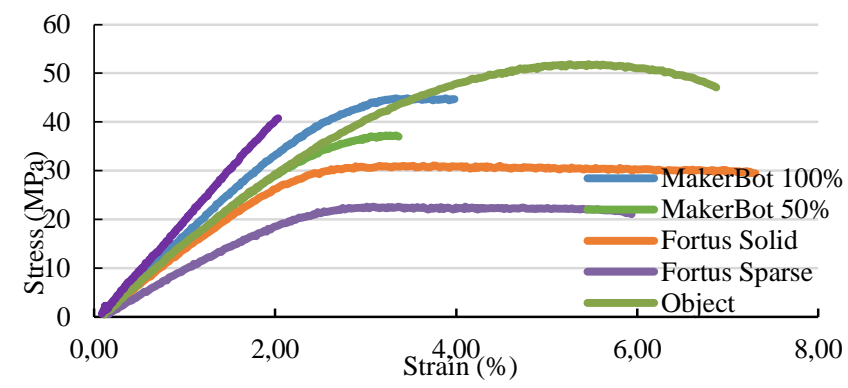

Fig. 6. Stress-strain curve comparison 


\section{Conclusions and Future Work}

In this paper, a case study of 3D print the linkage mechanism of a desktop robot arm was carried out. Among the three 3D printers applied in this paper, Object30 Prime had the best performance in terms of building time, dimensional accuracy, and end effect accuracy. MakerBot Replicator was the most material cost effective machine. Specimens of both Fortus and Object printers were better in dimensional accuracy and assembly accuracy than the original MeArm robot arm. All the hole diameters of 3D printing parts were smaller than the nominal size because of material shrinkage. Moreover, $3 \mathrm{D}$ printing parts were thicker than the nominal size in the $\mathrm{z}$ direction. The tensile test showed that VeroBlue had the largest yield strength and tensile strength. PLA part had larger tensile strength than ABS part and acrylic part, but it was not as ductile as ABS part. Acrylic part was the most brittle of the specimens. According to FEA simulation results, 3D printing specimens were able to meet the mechanical property requirements. For the FDM process, reducing interior density resulted in a slightly decrease in building time and material cost, but caused a significant decrease in yield strength.

Further research on devising an optimal way to adjust process parameters to meet a given set of requirements can be carried out.

\section{References}

1. Baich, L., Manogharan, G., Marie, H.: Study of Infill Print Design on Production Cost-time of 3D Printed ABS parts. Int. J. Rapid Manufacturing, 5(3-4), 308-319 (2015)

2. Davis, S. M.: From "Future Perfect": Mass customizing. Planning Rev., 17(2), 16-21 (1999)

3. Fogliatto, F. S., da Silveira, G. J., Borenstein, D.: The Mass Customization Decade: An Updated Review of the Literature. Int. J. Production Economics, 138(1), 14-25 (2012)

4. Kim, G. D., Oh, Y. T.: A Benchmark Study on Rapid Prototyping Processes and Machines. Proceedings of the Institution of Mechanical Engineers, 222(2), 201-215 (2008)

5. Kumar, A.: Mass Customization: Manufacturing Issues and Taxonomic Analyses. International Journal of Flexible Manufacturing Systems, 19(4), 625-629 (2007)

6. Lipson, H., Kurman, M.: Factory@ home: The Emerging Economy of Personal Fabrication. A report commissioned by the US Office of Science and Technology Policy (2010).

7. Mahesh, M., Wong, Y. S., Fuh, J. Y. H., Loh, H. T.: Benchmarking for Comparative Evaluation of RP Systems and Processes. Rapid Prototyping Journal, 10(2), 123-135 (2004)

8. Melenka, G. W., Schofield, J. S., Dawson, M. R., Carey, J. P.: Evaluation of Dimensional Accuracy and Material Properties Of The Makerbot 3D Desktop Printer. Rapid Prototyping Journal, 21(5), 618-627 (2015)

9. Mowatt, S.: Impacts of the Digital Economy. Idea Group, 136-153 (2005)

10. PolyJet Technology, http://www.stratasys.com/3d-printers/technologies/polyjet-technology

11. Salvador, F., De Holan, P. M., Piller, F. T.: Cracking the Code of Mass Customization. Sloan Management Review, 50(3), 71 (2009)

12. Sidambe, A. T Biocompatibility of Advanced Manufactured Titanium Implants: A Review. Materials, 7(12), 8168-8188 (2014)

13. Matter, http://my3dmatter.com/influence-infill-layer-height-pattern/ 